\title{
Antibacterial Performance of Alginic Acid Coating on Polyethylene Film
}

\author{
Elika Karbassi ${ }^{1}$, Ahmad Asadinezhad ${ }^{1}$, Marian Lehocký ${ }^{2, *}$, Petr Humpolíček ${ }^{2}$, Alenka Vesel ${ }^{3}$, \\ Igor Novák ${ }^{4}$ and Petr Sáha ${ }^{2}$
}

1 Department of Chemical Engineering, Isfahan University of Technology, Esfahan 84156-83111, Iran; E-Mails: e.karbassi@ce.iut.ac.ir (E.K.); asadinezhad@cc.iut.ac.ir (A.A.)

2 Centre of Polymer Systems, Tomas Bata University in Zlín, Zlín 76001, Czech Republic;

E-Mails: humpolicek@uni.utb.cz (P.H.); saha@utb.cz (P.S.)

3 Department of Surface Engineering, Jožef Stefan Institute, Ljubljana 1000, Slovenia;

E-Mail: alenka.vesel@ijs.si

4 Polymer Institute, Slovak Academy of Sciences, Bratislava 84236, Slovakia;

E-Mail: upolnovi@savba.sk

* Author to whom correspondence should be addressed; E-Mail: lehocky@post.cz;

Tel.: +420-608-616-048; Fax: +420-576-031-444.

Received: 9 June 2014; in revised form: 11 July 2014 / Accepted: 29 July 2014 /

Published: 21 August 2014

\begin{abstract}
Alginic acid coated polyethylene films were examined in terms of surface properties and bacteriostatic performance against two most representative bacterial strains, that is, Escherichia coli and Staphylococcus aureus. Microwave plasma treatment followed by brush formation in vapor state from three distinguished precursors (allylalcohol, allylamine, hydroxyethyl methacrylate) was carried out to deposit alginic acid on the substrate. Surface analyses via various techniques established that alginic acid was immobilized onto the surface where grafting (brush) chemistry influenced the amount of alginic acid coated. Moreover, alginic acid was found to be capable of bacterial growth inhibition which itself was significantly affected by the brush type. The polyanionic character of alginic acid as a carbohydrate polymer was assumed to play the pivotal role in antibacterial activity. The cell wall composition of two bacterial strains along with the substrates physicochemical properties accounted for different levels of bacteriostatic performance.
\end{abstract}


Keywords: alginic acid; polyethylene; surface modification; antibacterial activity; polysaccharide

\section{Introduction}

The surface features of biomaterials govern the interactions with biological molecules and play a decisive role in biofunctional materials development. This is why surface engineering has been practiced over recent decades to deal with a number of medical device related challenges [1]. Among numerous surface modification techniques, plasma is an effective route to activate soft matter surfaces having distinct advantages in terms of nanoscale changes on polymer surfaces while maintaining bulk properties intact [2-4]. Further stable enhancement of the surface reactivity can be achieved by grafting hydrophilic monomers of a vinyl type onto the surface of different substrates leading to brush formation, so that the resultant surfaces of materials reach the desired level of chemical functionality and characteristics for intended applications [5]. A functional brush not only provides desired chemistry for immobilization of bioactive entities but also prevents such entities from surface-induced denaturation of biomolecules [6]. The grafting-from approach has become the preferred option for the synthesis of polymer brushes where better control and higher reaction rate are achieved when the brush is developed in vapor phase. Viscosity in vapor state is not a determining factor, besides; there is less contamination as well as unwanted side reactions [7].

Bacterial infections are one of the critical scourges for mankind. Antibacterial agents based on biopolymers are important alternatives to low molecular weight biocides as they are usually non-toxic and can be used as effective surface coatings which inhibit bacterial proliferation [8]. Polysaccharides are polymeric carbohydrate molecules composed of long chains of monosaccharide units which are bound together by glycoside linkages. They possess versatile structural configurations and distinguished properties from their building blocks and have been studied for various biomedical applications [9,10]. Alginic acid is a carbohydrate polymer of great potential and a naturally occurring hydrophilic colloidal polysaccharide consisting mainly of residues of D-mannuronic acid and L-glucuronic acid obtained from varied species of brown seaweed [11]. It is an effective polyanion being readily associable with many molecules through ionic interactions or covalent bonds. Although there have been published many reports on alginic acid as a stand-alone material in the literature, there are few reports devoted to the alginic acid coatings on material surfaces intended for biomedical purposes [12-16], of which none of them has yet drawn their attention into its potential antibacterial activity. With regards to the prominent position of polyethylenes in the medical plastics arena $[17,18]$, and to provide new insights into the bacteriostatic potentials of alginic acid, the present novel effort is aimed at modifying low-density polyethylene (LDPE) films with an aim to impart an antibacterial property. In addition, the influence of three different graft types of allyalmine, allylalcohol, and hydroxyethyl methacrylate on surface properties and alginic acid coating quality is also explored through various surface analysis tools. 


\section{Results and Discussion}

To conveniently refer to the samples throughout the paper, each one is assigned a number as follows, sample 1: (untreated/control substrate), sample 2: plasma treated substrate, sample 3: allylalcohol (AAL) grafted substrate, sample 4: allylamine (AAM) grafted substrate, sample 5: 2-hydroxyethyl methacrylate (HEMA) grafted substrate, sample 6: alginic acid (ALGA) on AAL grafted substrate, sample 7: ALGA on AAM grafted substrate, and sample 8: ALGA on HEMA grafted substrate. The contact angle values of three testing liquids on untreated and modified substrates are given in Table 1. After plasma exposure, a reduction in contact angle values of three liquids on the sample surface is observed which suggests the enhanced hydrophilicity of the modified sample. Water and ethylene glycol droplets undergo further changes in terms of the angle they maintain with the solid surface compared with methylene iodide implying that polar component plays the major role in the interaction between droplet and the surface. This should result from the introduction of oxygen-containing moieties such as carbonyl, carboxyl, peroxide, and hydroperoxide on the surface. Further hydrophilicity is observed for samples 3-5 where the lowest water and ethylene glycol contact angle is exhibited by sample 5. Coating the substrate with ALGA diminishes the hydrophilicity, yet its level remains higher than that of sample 1. Such a reduction in hydrophilicity is most noticeable for sample 6. Since ALGA is hydrophilic in nature, the ALGA coated surface should typically have significant hydrophilicity. This is not clearly evident in the case of samples 6 and 7 as compared with sample 8, which could suggest that AAL and AAM are not as efficient grafts as HEMA brush. In other words, not only is HEMA more strongly bonded onto LDPE substrate, but also it shows higher reactivity towards ALGA, which facilitates coating and enhances quality of ALGA bonding as well as quantity of ALGA deposited.

Table 1. Contact angle $(\theta)$ analysis results of untreated and modified substrates using deionized water $(\mathrm{W})$, ethylene glycol $(\mathrm{E})$, and methylene iodide $(\mathrm{M})$ as wetting agents (contact angle data precision is indicated by standard deviation preceded by mean values).

\begin{tabular}{cccc}
\hline Sample No. & $\boldsymbol{\theta}_{\mathbf{W}}(\mathbf{0})$ & $\boldsymbol{\theta}_{\mathbf{E}}(\stackrel{ }{\circ})$ & $\boldsymbol{\theta}_{\mathbf{M}}(\mathbf{)}$ \\
\hline 1 & $92.5 \pm 6.1$ & $64.3 \pm 2.5$ & $45.8 \pm 2.3$ \\
2 & $51.5 \pm 20.2$ & $44.4 \pm 14.8$ & $42.9 \pm 23.7$ \\
3 & $39.4 \pm 5.5$ & $40.6 \pm 6.3$ & $50.9 \pm 6.7$ \\
4 & $38.8 \pm 6.0$ & $37.9 \pm 2.4$ & $50.3 \pm 4.6$ \\
5 & $35.7 \pm 4.3$ & $36.5 \pm 3.8$ & $55.9 \pm 2.6$ \\
6 & $76.2 \pm 6.7$ & $58.1 \pm 3.2$ & $52.6 \pm 5.0$ \\
7 & $63.0 \pm 7.3$ & $59.3 \pm 4.3$ & $54.0 \pm 5.0$ \\
8 & $56.0 \pm 6.4$ & $50.7 \pm 4.0$ & $63.2 \pm 8.2$ \\
\hline
\end{tabular}

Scanning electron microscope (SEM) images taken from untreated and modified substrates are illustrated in Figure 1. A relatively smooth, uniform morphology is well observed for sample 1 surface. Plasma treated substrate possesses quite distinct topography in terms of texture and roughness. This is normally expected after plasma in air as two simultaneous reactions (functionalization and ablation) take place as a consequence of energetic particle collision with surface molecules. Such morphology observed for sample 2 is favorable for subsequent grafting on account of increased surface area and free energy value. Upon exposing the plasma treated sample to monomer vapors (samples 3-5), 
a sensible change in topography comes about. Roughness slightly decreases and surface texture is significantly altered due to the strong effect of grafting on surface features. ALGA coating shows no considerable impact on surface morphology of AAL and AAM grafted substrates (samples 6 and 7) while a relatively smooth topography is seen for sample 8 . Bioactive molecules are supposed to play a filling influence on surface features resulting in a comparatively level morphology. It can point to the fact that the quantity of ALGA coated is significant for sample 8 compared with samples 6 and 7 . This can corroborate our contact angle analysis observation which says HEMA acts more efficiently for ALGA bonding.

Figure 1. Scanning electron microscope (SEM) micrographs of untreated and modified substrates (samples 1-8) taken at 30,000× magnification.
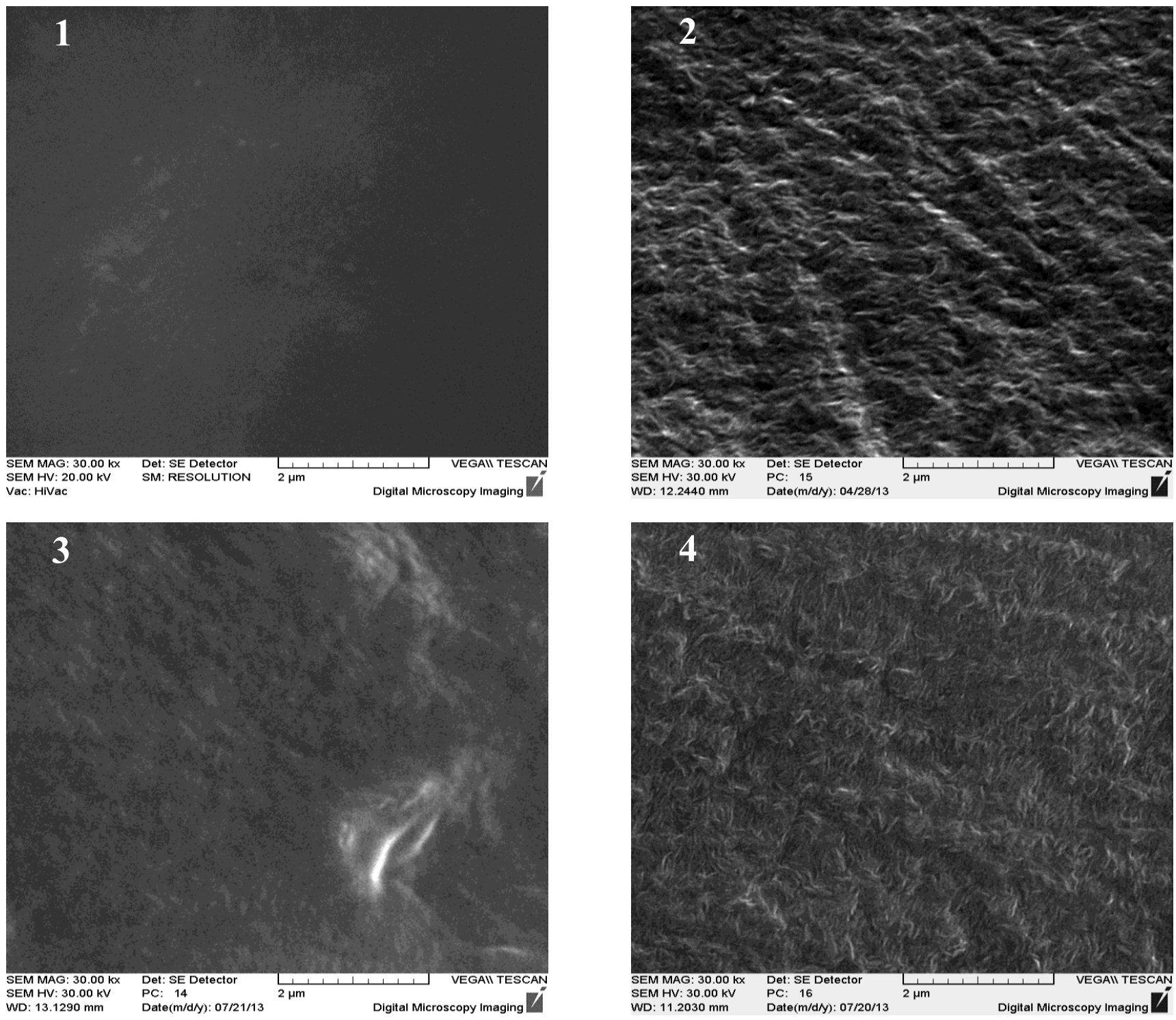
Figure 1. Cont.
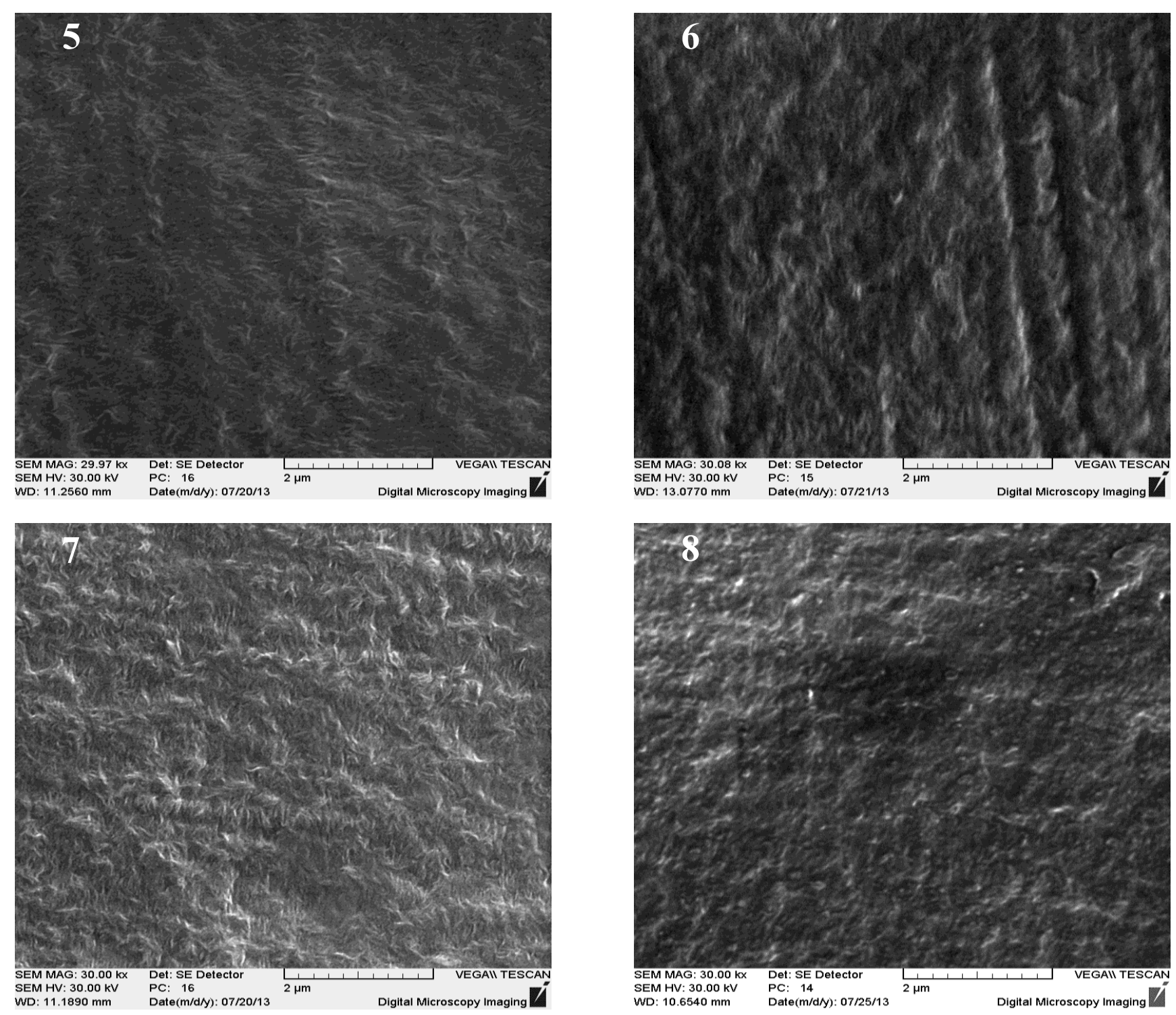

Attenuated total reflectance Fourier transform infrared (ATR-FTIR) spectra of untreated and modified substrates in absorption mode are illustrated in Figure 2. Three characteristic sets of major peaks within $650-2950 \mathrm{~cm}^{-1}$ resulting from $\mathrm{C}-\mathrm{H}$ bond vibrations in LDPE are easily seen, that is, one around $700 \mathrm{~cm}^{-1}$, the middle one at around $1500 \mathrm{~cm}^{-1}$ and a doublet peak around $3000 \mathrm{~cm}^{-1}$. They are all associated with various vibration modes of $\mathrm{C}-\mathrm{H}$ bond. Due to the high probe depth of ATR-FTIR, particularly at higher wavenumbers, no detectable change in the spectrum of sample 2 is observed compared with that of sample 1 except for some slight increase in peak intensities within $700-1500 \mathrm{~cm}^{-1}$ which can be assigned to $\mathrm{C}-\mathrm{O}$ and $\mathrm{C}-\mathrm{N}$ bonds vibration. As for sample 3, the LDPE characteristic peaks intensities are reduced, besides, the signal at around $1100 \mathrm{~cm}^{-1}$ strengthens in magnitude which is attributed to $\mathrm{C}-\mathrm{O}$ bond stretching in alcohols. Similar trends are also evident for sample 4 in which two broad peaks of minor magnitude, one at $1600 \mathrm{~cm}^{-1}$ and another around $1100 \mathrm{~cm}^{-1}$ assigned respectively to $\mathrm{N}-\mathrm{H}$ bending and $\mathrm{C}-\mathrm{N}$ stretching in amines are increased in magnitude. Sharper changes are evident for sample 5 where a broad peak at $1700 \mathrm{~cm}^{-1}$ associated with $\mathrm{C}=\mathrm{O}$ group is present together with a signal around $1100 \mathrm{~cm}^{-1}$ due to $\mathrm{C}-\mathrm{O}$ bond. Concerning samples 6 and 7 , no 
considerable changes arise in comparison with the spectra of samples 4 and 5, however; regarding sample 8 , obvious increase in $\mathrm{C}=\mathrm{O}$ and $\mathrm{C}-\mathrm{O}$ peaks intensity is noticeable. Moreover, a board peak within 3300-3500 $\mathrm{cm}^{-1}$ emerges ascribed to the hydroxyl group. The findings from FTIR analysis support observations from previous tests claiming that the HEMA graft is more favorable for ALGA coating and trace amounts of ALGA is coated on the AAL and AAM grafted samples so that they cannot be detected by ATR-FTIR probe.

Figure 2. Attenuated total reflectance Fourier transform infrared (ATR-FTIR) spectra of samples 1-8 within the entire range of wavenumber.

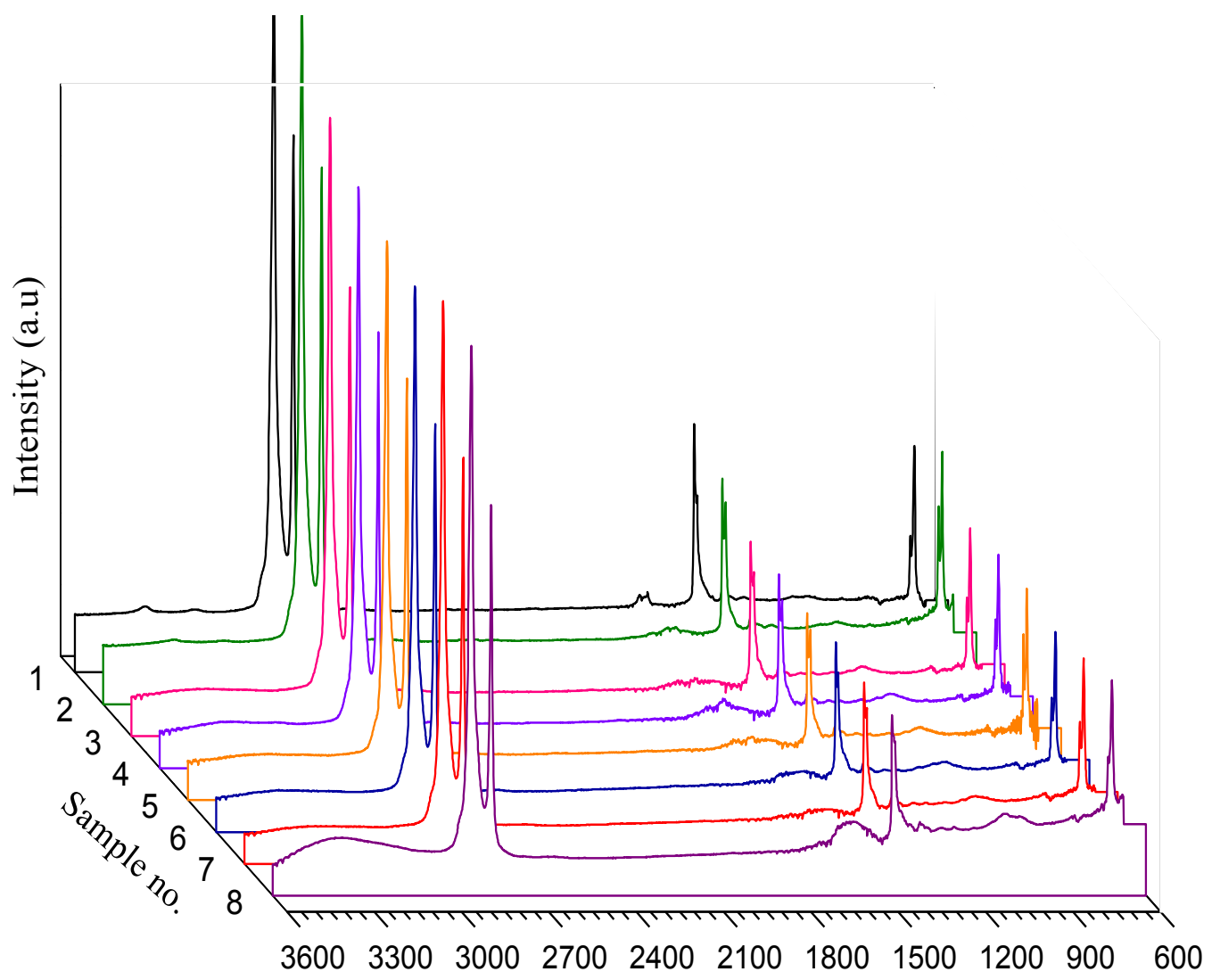

Wavenumber $\left(\mathrm{cm}^{-1}\right)$

Surface elemental compositions of untreated and modified substrates drawn out of the X-ray photoelectron spectroscopy (XPS) survey spectra are summarized in Table 2. Besides carbon, which is the main constituent, nitrogen and oxygen are also found in trivial amounts on sample 1, most likely due to additives or contaminants. A prominent increase in oxygen and nitrogen concentration at the expense of carbon content occurs regarding sample 2 due to the functionalization and ablation reactions after plasma exposure. Some slight changes in elemental concentrations are evident for sample 3 but not as much as expected since XPS probe depth is on the order of a few nanometers and may extend well beneath the brush. This can also indicate that the AAL layer thickness is not as high as expected most likely due to the low degree of polymerization. The same reasoning may be applied for sample 4, where an increase in nitrogen content and a reduction in oxygen concentration suggest the presence of an AAM layer on the surface, though of a very low thickness. In regard to sample 5, 
a reduction in nitrogen occurs as anticipated and the HEMA-grafted brush is not thick enough to extend beyond the XPS probe depth. A sharp decrease in oxygen content is observed after ALGA is coated onto the substrates (samples 6 and 7) while that of nitrogen varies depending on the graft type. Concerning sample 8 , oxygen is significantly increased while nitrogen quantity remains almost constant. It is then inferred that AAL and AAM are not as efficient grafts as the HEMA graft and in other words, not only is HEMA more strongly bonded onto the LDPE substrate, but also it favors ALGA coating. This finding supports the conclusion from previous analyses. The presence of calcium and chlorine in low amount on samples 6 and 7 is most likely due to the impurities present in purchased ALGA, however the significant amount of chlorine and manganese elements on the sample 8 surface is due to potassium permanganate and hydrogen chloride used after HEMA immobilization for carboxyl group activation.

Table 2. Elemental composition of untreated and modified substrate surfaces calculated from respective X-ray photoelectron spectroscopy (XPS) survey spectra (experimental absolute error is \pm 0.5 at.\%).

\begin{tabular}{ccccccc}
\hline Sample No. & C1s (at.\%) & O1s (at.\%) & N1s (at.\%) & C12p (at.\%) & Mn2p (at.\%) & Ca2p (at.\%) \\
\hline 1 & 99.0 & trace & trace & - & - & - \\
2 & 80.2 & 16.0 & 3.7 & - & - & - \\
3 & 81.2 & 15.8 & 3.0 & - & - & - \\
4 & 81.2 & 15.1 & 3.7 & - & - & - \\
5 & 81.8 & 15.2 & 3.0 & - & - & - \\
6 & 85.1 & 11.3 & 2.7 & 0.7 & - & trace \\
7 & 85.3 & 11.9 & 1.7 & 0.6 & - & 0.5 \\
8 & 68.5 & 20.3 & 2.8 & 5.1 & 1.1 & 2.1 \\
\hline
\end{tabular}

High resolution $\mathrm{C} 1 \mathrm{~s}$ signal of the samples are displayed in Figure 3. Modified substrates versus pristine sample have different $\mathrm{C} 1 \mathrm{~s}$ peak shapes that are directly connected with different chemical environments of the surface. A prominent peak is evident for samples 2-5 at around $289 \mathrm{eV}$ which corresponds to the $\mathrm{COOH}$ bond. A minor shoulder adjacent to the main peak is also evident for samples 6-8 around $286.5 \mathrm{eV}$ which is assigned to the $\mathrm{COH}$ group present in the ALGA molecule. This shoulder is meaningfully stronger for sample 8 giving credence to the aforementioned finding that HEMA acts more efficiently in coupling ALGA onto the surface.

The antibacterial capacity of the samples 1-8 based on the agar diffusion zone assay expressed as bacterial growth inhibition zone diameter are given in Table 3. Bacteriostatic agents limit the growth of bacteria by interfering with bacterial cellular metabolism, such as protein production and DNA replication. No characteristic inhibition zone is visible for samples 1-5 implying their bacteriostatic inability. As to samples 6-8, an adequate performance is evident against $E$. coli (gram negative) strain and to a lesser extent against the gram positive ( $S$. aureus) strain confirming bacteriostatic capability of ALGA-coated substrates. The graft type is interestingly found to affect the performance of the samples against bacteria where the highest activity against $E$. coli and the lowest against $S$. aureus are exhibited by sample 8 . The lowest activity against $E$. coli and the highest one against $S$. aureus are revealed by sample 7 and sample 6, respectively. As a matter of principle, net charge, hydrophilicity, and the amount of antibacterial agent deposited on the substrate affect the bacteriostatic quality. On the 
other hand, the cell wall structure and physicochemical characteristics of microorganisms are also of paramount importance. It is well recognized that $S$. aureus is different from E. coli in terms of cell wall composition which strongly influences the level of susceptibility. The former strain has a well-established cell wall made from a rigid peptidoglycan layer outside the cytoplasmic membrane, while the latter one possesses an outer cytoplasmic membrane made from lipopolysaccharide. Adherence of the bacteriostatic agent onto the outer cell wall and diffusivity into the membrane govern the vulnerability of the microorganism. It is therefore realized that ALGA can inhibit the bacterial growth due to its polyanionic character while coated onto a solid surface, whose level of effectiveness depends not only on the bacterial strain but also on the surface chemistry (graft type and bonding quality).

Figure 3. High resolution $\mathrm{C} 1 \mathrm{~s}$ peaks of samples 1-5 (top) and 6-8 (bottom) obtained from XPS analysis.
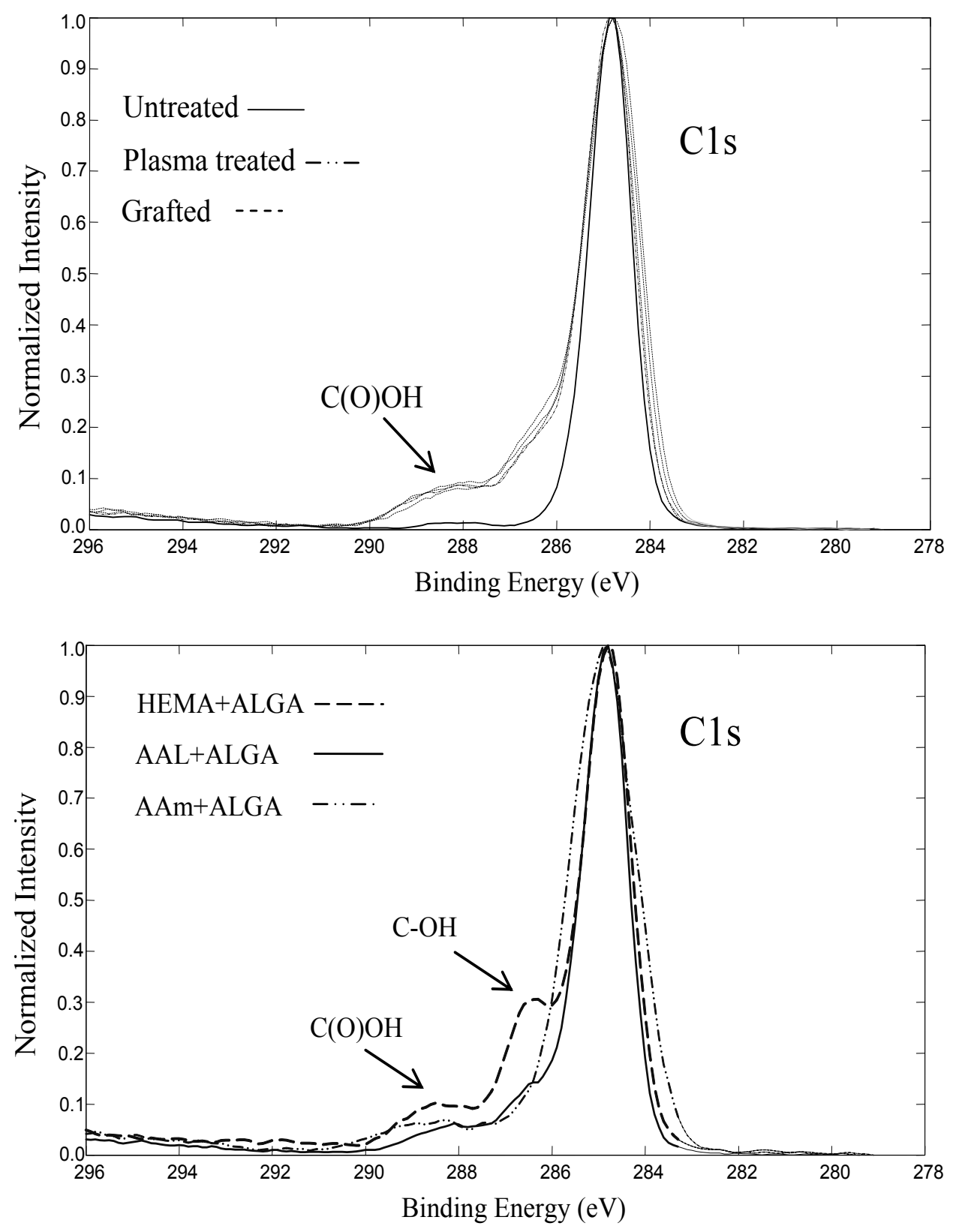
Table 3. Bacteriostatic performance of various substrates against two model bacteria represented by inhibition zone diameter (experimental percentage error is around $\pm 15 \%$ ).

\begin{tabular}{ccccccccc}
\hline Sample No. & $\mathbf{1}$ & $\mathbf{2}$ & $\mathbf{3}$ & $\mathbf{4}$ & $\mathbf{5}$ & $\mathbf{6}$ & $\mathbf{7}$ & $\mathbf{8}$ \\
\hline S. aureus & n.a & n.a & n.a & n.a & n.a & $14.0 \mathrm{~mm}$ & $10.0 \mathrm{~mm}$ & n.a \\
E. coli & n.a & n.a & n.a & n.a & n.a & $18.0 \mathrm{~mm}$ & $16.0 \mathrm{~mm}$ & $22.0 \mathrm{~mm}$ \\
\hline \multicolumn{6}{c}{ a: n.a stands for "not active" which implies that the substrate showed no measurable activity. }
\end{tabular}

\section{Experimental Section}

\subsection{Materials}

Low-density polyethylene film of $0.1 \mathrm{~mm}$ thickness was provided as packaging foil from Dow Chemicals, (Midland, MI, USA). Alginic acid from brown algae with 15\%-25\% carboxyl groups was provided from Sigma (St. Louis, MO, USA). Potassium permanganate, hydrochloric acid, 1-ethyl-3(3-dimethylaminopropyl) carbodiimide, allylamine, allylalcohol, and 2-hydroxyethyl methacrylate were obtained from Aldrich (St. Louis, MO, USA). The materials were used as received without prior treatment.

\subsection{Methods}

The surface modification approach adopted in this work was founded on our previously published papers [18-21], where a multistep procedure was followed to effectively coat the substrate with bioactive molecules (Figure 4). The procedure was principally designed in such a way which could immobilize the molecules on the surface through chemical bonds. LDPE foils were first cut into $5 \mathrm{~cm} \times 5 \mathrm{~cm}$ pieces, washed carefully with detergents to remove any surface pollutants, and fully dried in room conditions. The substrates were subjected to plasma treatment on both sides using a Pico Diener reactor (Ebhausen, Germany) operated at a pressure of $40 \mathrm{~Pa}$ in a microwave frequency of $2.45 \mathrm{GHz}$ with power of $50 \mathrm{~W}$ for $60 \mathrm{~s}$ to generate surface reactive groups. The carrier gas was air fed to the plasma chamber at the rate of 20 standard cubic centimeters per minute. Surface-activated substrates were immediately exposed to the saturated vapor of volatile monomers of AAM, AAL, and HEMA for $10 \mathrm{~s}$ at room temperature for polymeric brush formation. To convert the hydroxyl groups of HEMA-exposed substrate into activated carboxyl entities, the substrate was placed in $0.05 \mathrm{M}$ solution of potassium permanganate in hydrogen chloride for $1 \mathrm{~h}$ at $50{ }^{\circ} \mathrm{C}$, followed by immersion in $0.1 \% w / v$ aqueous solution of carbodiimide solution for $3 \mathrm{~h}$ at $4{ }^{\circ} \mathrm{C}$. Polysaccharide coating was subsequently carried out by immersing all substrates into $0.2 \mathrm{wt} \%$ ALGA aqueous solution of under constant shaking at $60 \mathrm{rpm}$ in room temperature for $24 \mathrm{~h}$. The modified substrates were finally taken out of solution, cleaned via ultrasonication with deionized water, dried, and stored in a dessicator for analysis. 
Figure 4. Alginic acid molecules coated onto polymeric brush spacer grown on plasma activated low-density polyethylene (LDPE) substrate.

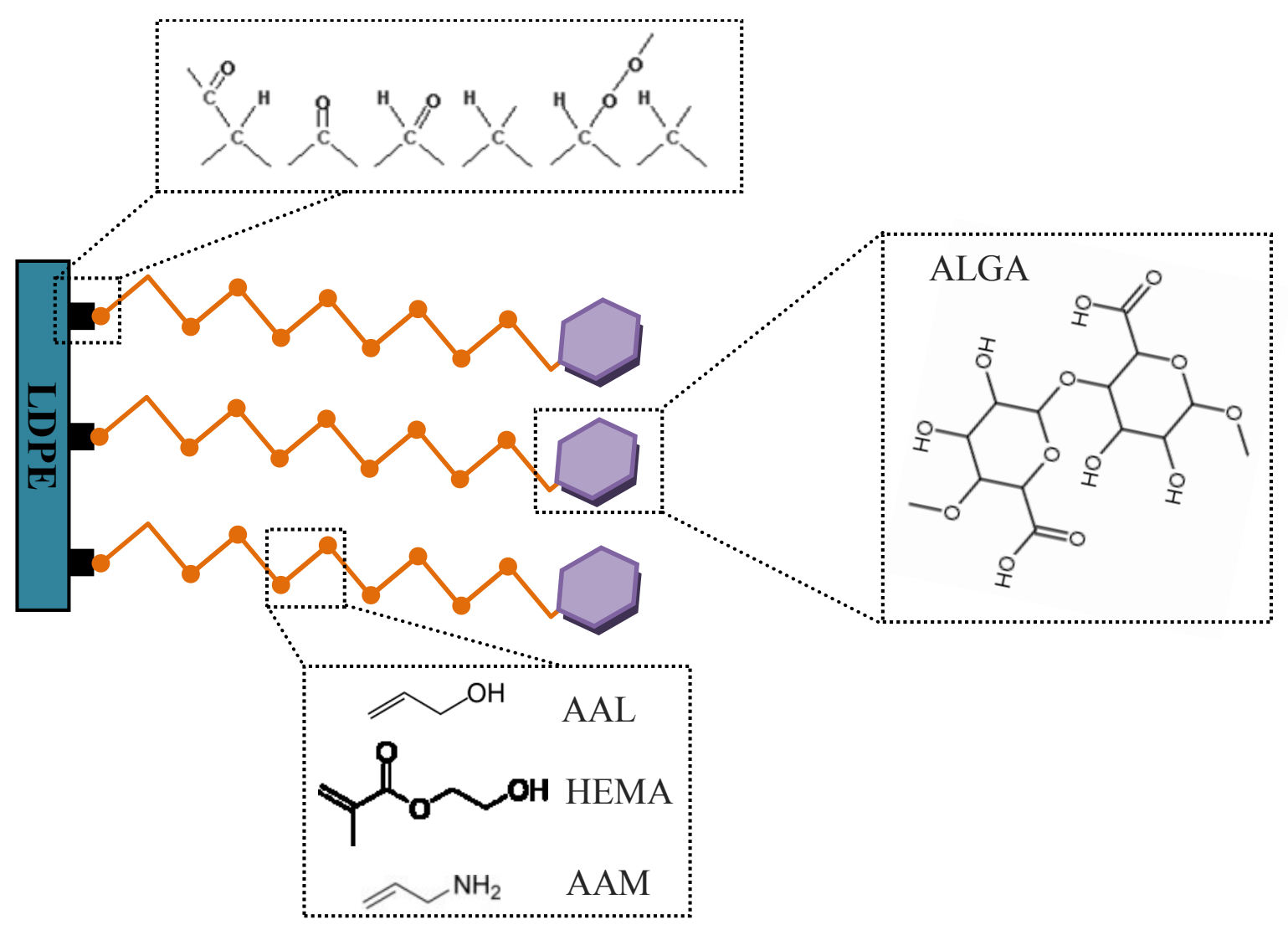

Static contact angle measurements for the surface energy estimation were performed by See System (Advex Instruments, Brno, Czech Republic) equipped with software using three standard testing liquids (water, ethylene glycol, and methylene iodide). Since water, ethylene glycol, and methylene iodide have different level of hydrophilicity, they have been employed for wettability evaluation. Water has the maximum surface tension in comparison with the other two liquids and ethylene glycol has the lowest (at room temperature). Surface tension is composed of two dispersive and polar components. Regarding water, the polar component plays the major role while in the case of ethylene glycol, the dispersive component is higher. As for methylene iodide, the polar component is zero and the whole surface tension is composed of dispersive element [22]. Sessile drop method was followed for all measurements where a $2 \mu \mathrm{L}$ drop was laid on each substrate in room temperature and the contact angle was visualized by a camera. An average of 10 measured values was reported. Attenuated total reflectance Fourier transform infrared (ATR-FTIR) spectroscopy was performed on Thermo Scientific Avatar 320 Nicolet (Waltham, MA, USA) at spectral resolution of $2 \mathrm{~cm}^{-1}$ for surface chemical characterization. The spectrometer was equipped with a $\mathrm{ZnSe}$ crystal at an incident angle of $45^{\circ}$. Each spectrum represents 64 co-added scans referenced against an empty ATR cell spectrum. X-ray photoelectron spectroscopy (XPS) was conducted using XPS Physical Electronics (Minneapolis, $\mathrm{MN}, \mathrm{USA})$. The base pressure in the XPS analysis chamber was about $6 \times 10^{-8} \mathrm{~Pa}$. The samples were

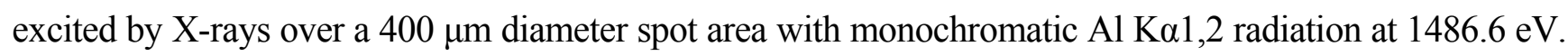
The emitted photoelectrons were detected by a hemispherical analyzer positioned at take-off angle of 
$45^{\circ}$. Survey-scan spectra were obtained at $187.85 \mathrm{eV}$ pass energy and $0.4 \mathrm{eV}$ step resolution. An electron gun was employed for surface neutralization. The elemental concentration analysis was performed over two different positions by the instrument software. High-resolution spectra of $\mathrm{C} 1 \mathrm{~s}$ were recorded at pass energy of 23.5 and $0.1 \mathrm{eV}$ energy step. Morphological changes were tracked by SEM, VEGA II LMU (Tescan, Brno, Czech Republic) at 30,000× magnification in high vacuum/secondary electron imaging mode at an accelerating voltage of $20 \mathrm{kV}$ for pristine LDPE foil and $30 \mathrm{kV}$ for modified samples. The substrates were sputter coated before analysis with a thin layer of palladium/gold alloy and tilted $15^{\circ}$ to attain qualitative insight into the level of roughness of surface features thanks to a stereoscopic view. In vitro antibacterial (bacteriostatic) activity was evaluated by agar diffusion method (Kirby-Bauer disk diffusion assay [23]) against two bacterial strains: Staphylococcus aureus, S. aureus 4516 (gram positive) and Escherichia coli, E. coli 4517 (gram negative) purchased from the Czech Collection of Microorganisms (Brno, Czech Republic). Circular pieces (disks) of $8 \mathrm{~mm}$ diameter were cut from $5 \mathrm{~cm} \times 5 \mathrm{~cm}$ substrates, washed and dried completely, and put on nutrient agar plate M1269 from HiMedia Laboratories (Mumbai, India) inoculated with bacterial suspension (volume: $100 \mu \mathrm{L}$, concentration: $10^{7}$ units $/ \mathrm{mL}$ ). After incubation time of $24 \mathrm{~h}$ in $37{ }^{\circ} \mathrm{C}$, the inhibition zone diameter was measured in five directions and the averaged value was reported. Each test was repeated in triplicate and the experimental error was calculated.

\section{Conclusions}

In this effort, low-density polyethylene films have been surface modified through microwave plasma treatment followed by grafting polymerizable monomers to provide appropriate functionalities needed for alginic acid coating. Alginic acid has been found to be effective against bacterial growth inhibition (gram positive and gram negative strains) and the level of activity depends strongly on grafting chemistry. 2-Hydroxyethyl methacrylate has been determined to be a good choice for alginic acid binding compared to allylalcohol and allyalmie, and alginic acid has been shown to be coated onto the modified surface through chemical interactions, However, only in the case of 2-hydroxyethyl methacrylate can primary covalent bonds form, and regarding allylalcohol and allylamine, secondary or ionic interactions are most likely responsible for alginic acid immobilization. The obtained findings are of value from both basic science and applied standpoints. The current effort can also underlie the forthcoming attempts to realize interactions between alginic acid and pathogenic microorganisms. From an applied viewpoint, alginic acid coatings on polymer surfaces can be useful for single-use disposable medical devices where bacterial growth inhibition is required.

\section{Acknowledgments}

This work was supported by Operational Program Research and Development for Innovations co-funded by the European Regional Development Fund (ERDF) and national budget of Czech Republic, within the framework of project "Centre of Polymer Systems" (reg. number: CZ.1.05./2.1.00/03.0111). The authors also thank the Grant Agency of Czech Republic (GA13-08944S) and Ministry of Education of the Slovakia and Slovak Academy of Sciences project (Grant No. 2/0199/14) for financial support. 


\section{Author Contributions}

Experimental design: Ahmad Asadinezhad, Marian Lehocký; Sample Preparation: Elika Karbassi, Marian Lehocký; Contributions to materials/analysis tools: Petr Humpolíček, Alenka Vesel, Igor Novák, Petr Sáha; Manuscript preparation: Elika Karbassi, Ahmad Asadinezhad.

\section{Conflicts of Interest}

The authors declare no conflict of interest.

\section{References}

1. Goddard, J.M.; Hotchkiss, J.H. Polymer surface modification for the attachment of bioactive compounds. Prog. Polym. Sci. 2007, 32, 698-725.

2. Chu, P.K.; Chen, J.Y.; Wang, L.P.; Huang, N. Plasma-surface modification of biomaterials. Mater. Sci. Eng. 2002, 36, 143-206.

3. Desmet, T.; Morent, R.; de Geyter, N.; Leys, C.; Schacht, E.; Dubruel, P. Nonthermal plasma technology as a versatile strategy for polymeric biomaterials surface modification: A review. Biomacromolecules 2007, 10, 2351-2378.

4. Mozetic, M.; Ostrikov, K.; Ruzic, D.N.; Curreli, D.; Cvelbar, U.; Vesel, A.; Primc, G.; Leisch, M.; Jousten, K.; Malyshev, O.B.; et al. Recent advances in vacuum sciences and applications. J. Phys. D: Appl. Phys. 2014, 47, 153001-153023.

5. Bhattacharya, A.; Misra, B.N. Grafting: A versatile means to modify polymers techniques, factors and applications. Prog. Polym. Sci. 2004, 29, 767-814.

6. Zhao, B.; Brittain, W.J. Polymer brushes: Surface-immobilized macromolecules. Prog. Polym. Sci. 2000, 25, 677-710.

7. Ayres, N. Polymer brushes: Applications in biomaterials and nanotechnology. Polym. Chem. 2010, 1, 769-777.

8. Waschinski, C.J. Tiller, J.C. Poly(oxazoline)s with telechelic antimicrobial functions. Biomacromolecules 2005, 6, 235-243.

9. Elsabee, M.Z.; Abdou, E.S.; Nagy, K.S.A.; Eweis, M. Surface modification of polypropylene films by chitosan and chitosan/pectin multilayer. Carbohydr. Polym. 2008, 71, 187-195.

10. El-tahlawy, K.F.; El-bendary, M.A.; El-hendawy, A.G.; Hudson, S.M. The antimicrobial activity of cotton fabrics treated with different crosslinking agents and chitosan. Carbohydr. Polym. 2005, $60,421-430$.

11. Ikeda, A.; Takemura, A.; Ono, H. Preparation of low-molecular weight alginic acid by acid hydrolysis. Carbohydr. Polym. 2000, 42, 421-425.

12. Yoshioka, T.; Tsuru, K.; Hayakawa, S.; Osaka, A. Preparation of alginic acid layers on stainless-steel substrates for biomedical applications. Biomaterials 2003, 24, 2889-2894.

13. Morra, M.; Cassinelli, C. Simple model for the XPS analysis of polysaccharide-coated surfaces. Surf. Interface Anal. 1998, 26, 742-746.

14. Morra, M.; Cassinelli, C. Surface studies on a model cell-resistant system. Langmuir 1999, 15, 4658-4663. 
15. Morra, M.; Cassinelli, C. Non-fouling properties of polysaccharide-coated surfaces. J. Biomater. Sci-Polym. E 1999, 10, 1107-1124.

16. Morra, M.; Cassinelli, C. Force measurements on cell repellant and cell adhesive alginic acid coated surfaces. Colloid Surf. B 2000, 18, 249-259.

17. Bilek, F.; Krizova, T.; Lehocky, M. Preparation of active antibacterial LDPE surface through multistep physicochemical approach: I. Allylamine grafting, attachment of antibacterial agent and antibacterial activity assessment. Colloid Surf. B 2011, 88, 440-447.

18. Bilek, F.; Sulovska, K.; Lehocky, M.; Saha, P.; Humpolicek, P.; Mozetic, M.; Junkar, I. Preparation of active antibacterial LDPE surface through multistep physicochemical approach II: Graft type effect on antibacterial properties. Colloid Surf. B 2013, 102, 842-848.

19. Asadinezhad, A.; Novak, I.; Lehocky, M.; Sedlarik, V.; Vesel, A.; Junkar, I.; Saha, P.; Chodak, I. A physicochemical approach to render antibacterial surfaces on medical-grade PVC. Plasma Process. Polym. 2010, 7, 504-514.

20. Asadinezhad, A.; Novak, I.; Lehocky, M.; Sedlarik, V.; Vesel, A.; Junkar, I.; Saha, P.; Chodak, I. An in vitro bacterial adhesion assessment of surface modified medical-grade PVC. Colloid Surf. B 2010, 77, 246-256.

21. Asadinezhad, A.; Novak, I.; Lehocky, M.; Bilek, F.; Vesel, A.; Junkar, I.; Saha, P.; Popelka, A. Polysaccharide coatings on medical-grade PVC: A probe into surface characteristics and bacterial adhesion extent. Molecules 2010, 15, 1007-1027.

22. Fan, C.W.; Lee, S.C. Surface free energy effects in sputter-deposited $\mathrm{WN}_{x}$ films. Mater. Trans. 2007, 48, 2449-2453.

23. Parija, S.C. Textbook of Microbiology \& Immunology; Elsevier: Amsterdam, The Netherlands, 2009; pp. 71-74.

(C) 2014 by the authors; licensee MDPI, Basel, Switzerland. This article is an open access article distributed under the terms and conditions of the Creative Commons Attribution license (http://creativecommons.org/licenses/by/3.0/). 